\title{
Cyclical contractive conditions in probabilistic metric spaces
}

\author{
Abderrahim Mbarki $]^{1}$, Rachid Oubrahim ${ }^{2}$ \\ ${ }^{1}$ ANO Laboratory, National School of applied sciences, Oujda university, P.O. BOX 669, Morocco \\ ${ }^{2}$ ANO Laboratory, Faculty of sciences, Oujda university, 60000, Morocco
}

\begin{tabular}{l} 
A R T I C L E I N F O \\
\hline Article history: \\
Received: 10 June, 2017 \\
Accepted: 15 July, 2017 \\
Online: 28 December, 2017 \\
\hline Keywords: \\
Probabilistic metric spaces \\
Cyclic contractions \\
Fixed point \\
Probabilistic k-contractions
\end{tabular}

\section{Introduction}

Fixed points theory plays a basic role in applications of many branches of mathematics. Finding a fixed point of contractive mappings becomes the center of strong research activity. After that, based on this finding, a large number of fixed point results have appeared in recent years. Generally speaking, there usually are two generalizations on them, one is from spaces, the other is from mappings.

Concretely, for one thing, from spaces, for example, the concept of a probabilistic metric spaces was introduced in 1942 by Karl Menger [1], indeed, he proposed replacing the distance $d(p, q)$ by a real function $F_{p q}$ whose value $F_{p q}(x)$ for any real number $x$ is interpreted as the probability that the distance between $p$ and $q$ is less than $x$.

For another thing, from mappings, for instance, let $A$ and $B$ be nonempty subsets of a metric space $(M, d)$ and let $f: A \cup B \rightarrow A \cup B$ be a mapping such that:

(1) $f(A) \subseteq B$ and $f(B) \subseteq A$.

(2) $d(f x, f y) \leq k d(x, y), \forall x \in A, \forall y \in B$, where $k \in[0,1)$.

If (1) holds we say that $f$ is a cyclic map and if (1) and

(2) hold we say that $f$ is a cyclic contraction [2].

In this work, we show the existence and uniqueness of the fixed point for the cyclic probabilistic $k$-contraction mapping in a probabilistic metric spaces.

\section{Preliminaries}

Throughout this work, we adopt the usual terminology, notation and conventions of the theory of probabilistic metric spaces, as in [3].

Definition 2.1. A distance distribution function (briefly, a d.d.f.) is a nondecreasing function $F$ defined on $\mathbb{R}^{+} \cup\{\infty\}$ that satisfies $f(0)=0$ and $f(\infty)=1$, and is left continuous on $(0, \infty)$. The set of all d.d.f's will be noted by $\Delta^{+}$; and the set of all F in $\Delta^{+}$for which $\lim _{t \rightarrow \infty} f(t)=1$ by $D^{+}$.

For any $a$ in $\mathbb{R}^{+} \cup\{\infty\}, \varepsilon_{a}$, the unit step at $a$, is the function given by:

for $0 \leq a<\infty$

$$
\varepsilon_{a}(x)=\left\{\begin{array}{lll}
0 & \text { if } & 0 \leq x \leq a \\
1 & \text { if } & a<x \leq \infty
\end{array}\right.
$$

and

$$
\varepsilon_{\infty}(x)=\left\{\begin{array}{ccc}
0 & \text { if } & 0 \leq x \leq \infty \\
1 & \text { if } & x=\infty
\end{array}\right.
$$

Note that $\varepsilon_{a} \leq \varepsilon_{b}$ if and only if $b \leq a$; that $\varepsilon_{a}$ is in $D^{+}$if $0 \leq a<\infty$; and that $\epsilon_{0}$ is the maximal element, and $\epsilon_{\infty}$ the minimal element, of $\Delta^{+}$.

Definition 2.2. Consider $f$ and $g$ be in $\Delta^{+}, h \in(0,1]$, and let $(f, g ; h)$ denotes the condition

$$
0 \leq g(x) \leq f(x+h)+h,
$$

for all $x$ in $\left(0, \frac{1}{h}\right)$.

The modified Levy distance is the function $d_{L}$ defined on

${ }^{*}$ Corresponding Author: Abderrahim Mbarki, NATIONAL SCHOOL OF APPLIED SCIENCES, P.O. BOX 669, OUJDA UNIVERSITY. Email: dr.mbarki@gmail.com 
$\Delta^{+} \times \Delta^{+} b y$

$d_{L}(f, g)=\inf \{h$ : both conditions $(f, g ; h)$ and $(g, f ; h)$ hold\}.

Note that for any $f$ and $g$ in $\Delta^{+}$, both $(f, g ; 1)$ and $(g, f ; 1)$ hold, hence $d_{L}$ is well-defined and $d_{L}(f, g) \leq 1$.

Lemma 2.1. The function $d_{L}$ is a metric on $\Delta^{+}$.

Definition 2.3. A sequence $\left\{F_{n}\right\}$ of d.d.f's is said to converge weakly to a d.d.f. $F$ if and only if the sequence $\left\{F_{n}(x)\right\}$ converges to $F(x)$ at each continuity point $x$ of $F$.

Lemma 2.2. Let $\left\{F_{n}\right\}$ be a sequence of functions in $\Delta^{+}$, and let $F$ be in $\Delta^{+}$. Then $\left\{F_{n}\right\}$ converges weakly to $F$ if and only if $d_{L}\left(F_{n}, F\right) \rightarrow 0$.

Lemma 2.3. The metric spaces $\left(\Delta^{+}, d_{L}\right)$ is compact, and hence complete.

Lemma 2.4. For any $F$ in $\Delta^{+}$and $t>0$,

$$
F(t)>1-t \text { iff } d_{L}\left(F, \varepsilon_{0}<t\right) .
$$

Lemma 2.5. If $F$ and $G$ are in $\Delta^{+}$and $F \leq G$ then $d_{L}\left(G, \varepsilon_{0}\right) \leq d_{L}\left(F, \varepsilon_{0}\right)$.

Definition 2.4. A triangular norm (briefly, a t-norm) is a binary operation $T$ on $[0,1]$ such that:

$$
\begin{aligned}
T(x, y) & =T(y, x), \quad(\text { commutativity) } \\
T(x, y) & \leq T(z, w), \text { whenever } x \leq z, y \leq w, \\
T(x, 1) & =x,(1 \text { is an identity element) } \\
T(T(x, y), z) & =T(x, T(y, z)),(\text { associativity). }
\end{aligned}
$$

Example 2.1. The following $t$-norms are continuous:

(i) The t-norm minimum $M(x, y)=\operatorname{Min}(x, y)$.

(ii) The $t$-norm product $\prod(x, y)=x y$.

(iii) The t-norm $W, W(x, y)=\operatorname{Max}(x+y-1,0)$.

Definition 2.5. A triangle function is a binary operation $\tau$ on $\Delta^{+}$that is commutative, associative, and nondecreasing in each place, and has $\varepsilon_{0}$ as identity.

Example 2.2. If $T$ is left continuous, then the binary operation $\tau_{T}$ on $\Delta^{+}$defined by:

$$
\tau_{T}(F, G)(x)=\sup \{T(F(u), G(v)): u+v=x\}
$$

is a triangle function.

Lemma 2.6. If $T$ is continuous, then $\tau_{T}$ is continuous .

Definition 2.6. A probabilistic metric space (briefly a bms $)$ is a triple $(M, F, \tau)$ where $M$ is a nonempty set, $F$ is a function from $M \times M$ into $\Delta^{+}, \tau$ is a triangle function, and the following conditions are satisfied

for all $p, r ; q \in S$

(i) $F_{p p}=\varepsilon_{0}$,

(ii) $F_{p r}=\varepsilon_{0} \Rightarrow p=r$,

(iii) $F_{p r}=F_{r p}$

(iv) $F_{p r} \geq \tau\left(F_{p q}, F_{q r}\right)$.

If $\tau=\tau_{T}$ for some t-norm $T$, then $\left(M, F, \tau_{T}\right)$ is called a Menger space.
It should be noted that if $T$ is a continuous $t$-norm, then $(M, F)$ satisfies $(i v)$ under $\tau_{T}$ if and only if it satisfies

$$
\text { (v) } F_{p r}(x+y) \geq T\left(F_{p q}(x), F_{q r}(y)\right)
$$

for all $p, r ; q \in M$ and for all $x, y>0$, under $T$.

Definition 2.7. Let $(M, F)$ be a probabilistic semimetric space (i.e., (i), (ii) and (iii) of Definition 2.6 are satisfied). For $p$ in $M$ and $t>0$, the strong $t$-neighborhood of $p$ is the set

$$
N_{p}(t)=\left\{q \in M: F_{p q}(t)>1-t\right\} .
$$

The strong neighborhood system at $p$ is the collection $\wp_{p}=\left\{N_{p}(t): t>0\right\}$, and the strong neighborhood system for $M$ is the union $\wp=\bigcup_{p \in M} \wp_{p}$.

An immediate consequence of Lemma 2.4 is

$$
N_{p}(t)=\left\{q \in M: d_{L}\left(F_{p q}, \varepsilon_{0}\right)<t\right\} .
$$

In probabilistic semimetric space, the convergence of sequence is defined in the way

Definition 2.8. Let $\left\{x_{n}\right\}$ be a sequence in a probabilistic semimetric space $(M, F)$. Then

(1) The sequence $\left\{x_{n}\right\}$ is said to be convergent to $x \in M$, if for every $\epsilon>0$, there exists a positive integer $N(\epsilon)$ such that $F_{x_{n} x}(\epsilon)>1-\epsilon$ whenever $n \geq N(\epsilon)$.

(2) The sequence $\left\{x_{n}\right\}$ is called a Cauchy sequence, if for every $\epsilon>0$ there exists a positive integer $N(\epsilon)$ such that $n, m \geq N(\epsilon) \Rightarrow F_{x_{n} x_{m}}(\epsilon)>1-\epsilon$.

(3) $(M, F)$ is said to be complete if every Cauchy sequence has a limit. duce.

The proof of the following result is easy to repro-

Proposition 2.1. Let $\left\{x_{n}\right\}$ be a sequence in a probabilistic semimetric space $(M, F)$ and $x \in M$.

$1-\left\{x_{n}\right\}$ is convergent to $x$, if either

- $\lim _{n \rightarrow \infty} F_{x_{n} x}(t)=1$ for all $t>0$, or

- for every $\epsilon>0$ and $\delta \in(0,1)$, there exists a positive integer $N(\epsilon, \delta)$ such that $F_{x_{n} x}(\epsilon)>1-\delta$, whenever $n \geq N(\epsilon, \delta)$.

$2-\left\{x_{n}\right\}$ is Cauchy sequence, if either

- $\lim _{n, m \rightarrow \infty} F_{x_{n} x_{m}}(t)=1$ for all $t>0$, or

- for every $\epsilon>0$ and $\delta \in(0,1)$, there exists a positive integer $N(\epsilon, \delta)$ such that $F_{x_{n} x_{m}}(\epsilon)>1-\delta$, whenever $n, m \geq N(\epsilon, \delta)$.

Scheizer and Sklar [3] proved that if $(M, F, \tau)$ is a probabilistic metric space with $\tau$ is continuous, then the family I consisting of $\emptyset$ and all unions of elements of this strong neighborhood system for $M$ determines a Hausdorff topology for $M$.

Consequently, in such space we have the following assertions

(a) $(M, F, \tau)$ is endowed with the topology I is a Hausdroff topological space.

(b) There exists a topology $\Lambda$ on $S$ such that the strong neighborhood system $\wp$ is a basis for $\Lambda$. 
Let $f$ a self map on $M$. Power of $f$ at $p \in M$ are defined by $f^{0} p=p$ and $f^{n+1} p=f\left(f^{n} p\right), n \geq 0$. We will use the notation $p_{n}=f^{n} p$, in particular $p_{0}=p$, $p_{1}=f p$.

The letter $\Psi$ denotes the set of all function $\varphi$ : $[0, \infty) \rightarrow[0, \infty)$ such that

$$
0<\varphi(t)<t \text { and } \lim _{n \rightarrow \infty} \varphi^{n}(t)=0 \text { for each } t>0
$$

Definition 2.9. [4] We say that a t-norm $T$ is of H-type if the family $\left\{T^{n}(t)\right\}$ is equicontinuous at $t=1$, that is,

$\forall \epsilon \in(0,1), \exists \lambda \in(0,1): t>1-\lambda \Rightarrow T^{n}(t)>1-$ $\epsilon, \forall n \geq 1$

Where $T^{1}(x)=T(x, x), T^{n}(x)=T\left(x, T^{n-1}(x)\right)$, for every $n \geq 2$. type.

The t-norm $T_{M}$ is a trivial example of t-norm of $\mathrm{H}$ -

Definition 2.10. [5] Let $\varphi:[0, \infty) \rightarrow[0, \infty)$ be a function such that $\varphi(t)<t$ for $t>0$, and $f$ be a selfmap of a probabilistic metric space $(M, F, \tau)$. We say that $f$ is $\varphi$-probabilistic contraction if

$$
F_{f p f q}(\varphi(t)) \geq F_{p q}(t)
$$

for all $p, q \in M$ and $t>0$,

Theorem 2.1. [6] Let $\left(M, F, \tau_{T}\right)$ be a complete probabilistic metric space under a continuous t-norm $T$ of $H$ type such that RanF $\subset D^{+}$. Let $f: M \rightarrow M$ be a $\varphi$ probabilistic contraction where $\varphi \in \Psi$. Then $f$ has a unique fixed point $\bar{x}$, and, for any $x \in M, \lim _{n \rightarrow \infty} f^{n}(x)=\bar{x}$.

\section{Cyclical contractive conditions in probabilistic metric spaces}

Theorem 3.1. Let $\left(M, F, \tau_{T}\right.$, ) be a complete probabilistic metric space under a continuous t-norm $T$ of $H$-type such that RanF $\subset D^{+}$. Let $f: M \rightarrow M$ be a continuous mapping and satisfies

$$
F_{f p f^{2} p}(k t) \geq F_{p f p}(t)
$$

for all $p \in M$ and $t>0$ where $k \in(0,1)$.

Then $f$ has a fixed point in $M$.

Proof. Let $p_{0} \in M$. Put $p_{n}=f\left(p_{n-1}\right)=f^{n}\left(p_{0}\right)$ for each $n \in\{0,1,2, \ldots\}$. We prove that $\left\{p_{n}\right\}$ is a Cauchy sequence in $M$. We need to show that for each $\delta>0$ and $0<\epsilon<1$ there exists a positive integer $n_{1}=n_{1}(\delta, \epsilon)$ such that:

$$
F_{p_{n} p_{m}}(\delta)>1-\epsilon \text { for all } m>n>n_{1}(\delta, \epsilon)
$$

For each $\delta>0$, for $m>n$ we have

$$
\begin{aligned}
F_{p_{n} p_{m}}(\delta) & \geq T\left(F_{p_{n} p_{n+1}}(\delta-k \delta), F_{p_{n+1} p_{m}}(k \delta)\right) \\
& \geq T\left(F_{p_{n-1} p_{n}}\left((\delta-k \delta) k^{-1}\right), F_{p_{n+1} p_{m}}(k \delta)\right) \\
& \geq T\left(F_{p_{n-2} p_{n-1}}\left((\delta-k \delta) k^{-2}\right), F_{p_{n+1} p_{m}}(k \delta)\right) \\
& \geq T\left(F_{p_{n-3} p_{n-2}}\left((\delta-k \delta) k^{-3}\right), F_{p_{n+1} p_{m}}(k \delta)\right) \\
& \vdots \\
& \geq T\left(F_{p_{0} p_{1}}\left((\delta-k \delta) k^{-n}\right), F_{p_{n+1} p_{m}}(k \delta)\right)
\end{aligned}
$$

It follows that

$F_{p_{n} p_{m}}(\delta) \geq T\left(F_{p_{0} p_{1}}\left((\delta-k \delta) k^{-n}\right), T\left(F_{p_{n+1} p_{n+2}}(k \delta-\right.\right.$ $\left.\left.\left.k^{2} \delta\right), F p_{n+2} p_{m}\left(k^{2} \delta\right)\right)\right)$

Then

$F_{p_{n} p_{m}}(\delta) \geq T\left(F_{p_{0} p_{1}}\left((\delta-k \delta) k^{-n}\right), T\left(F_{p_{n} p_{n+1}}(\delta-\right.\right.$ $\left.\left.k \delta), F_{p_{n+2} p_{m}}\left(k^{2} \delta\right)\right)\right)$

Then

$F_{p_{n} p_{m}}(\delta) \geq T\left(F_{p_{0} p_{1}}\left((\delta-k \delta) k^{-n}\right), T\left(F_{p_{n-1} p_{n}}((\delta-\right.\right.$ $\left.\left.\left.k \delta) k^{-1}\right), F p_{n+2} p_{m}\left(k^{2} \delta\right)\right)\right)$

Then

$F_{p_{n} p_{m}}(\delta) \geq T\left(F_{p_{0} p_{1}}\left((\delta-k \delta) k^{-n}\right), T\left(F_{p_{0} p_{1}}((\delta-\right.\right.$ $\left.\left.\left.k \delta) k^{-n}\right), F_{p_{n+2} p_{m}}\left(k^{2} \delta\right)\right)\right)$

Using the same argument repeatedly and by definition of the operator $T^{(n)}$, we obtain

$$
F_{p_{n} p_{m}}(\delta) \geq T^{m-n}\left(F_{p_{0} p_{1}}\left((\delta-k \delta) k^{-n}\right)\right)
$$

Since $T$ is a $t$-norm of $H$-type, for given $\lambda \in(0,1)$, there exists $\lambda=\lambda(\epsilon) \in(0,1)$ such that if we have $t>1-\lambda$ then $T^{n}(t)>1-\epsilon$ for all $n \geq 1$.

Since

$$
(\delta-k \delta) k^{-n} \rightarrow 0 \text { as } n \rightarrow \infty
$$

Then

$$
F_{p_{0} p_{1}}\left((\delta-k \delta) k^{-n}\right) \rightarrow 1 \text { as } n \rightarrow \infty
$$

because $F_{p_{0} p_{1}} \in D^{+}$.

Then there exists $N \in \mathbb{N}$ such that

$$
F_{p_{0} p_{1}}\left((\delta-k \delta) k^{-n}\right)>1-\lambda \text { for all } n>N(\lambda(\epsilon))
$$

Hence and by (3.1) we conclude that

$$
F_{p_{n} p_{m}}(\delta)>1-\epsilon \text { for all } m>n>N
$$

Thus we proved that the $\left\{p_{n}\right\}$ is a Cauchy sequence in M.

Since $M$ is complete there is some $q \in M$ such that

$$
p_{n} \rightarrow q
$$

The continuity of mapping $f$ and the uniqueness of the limit implies that

$$
f(q)=q
$$

We now state the main fixed point theorem for cyclical contractive conditions. 
Theorem 3.2. Let $\left(M, F, \tau_{T}\right.$, be a complete probabilistic metric space under a continuous t-norm $T$ of $H$-type such that RanF $\subset D^{+}$. Let $A$ and $B$ be nonempty closed subsets of $M$ and let $f: A \cup B \rightarrow A \cup B$ be a mapping and satisfies: (1) $F(A) \subset B$ and $F(B) \subset A$.

(2) $F_{f p f q}(k t) \geq F_{p q}(t), \forall p \in A$ and $\forall q \in B$, where $k \in$ $(0,1)$.

Then $f$ has a unique fixed point in $A \cap B$.

Proof. For $p \in A \cup B$ we have

$$
F_{f p f^{2} p}(k t) \geq F_{p f p}(t)
$$

By theorem $3.1\left\{p_{n}\right\}$ is a Cauchy sequence. Consequently $\left\{p_{n}\right\}$ converges to some point $q \in M$. However in view of (2) an infinite number of terms of the sequence $\left\{p_{n}\right\}$ lie in $A$ and an infinite number of terms lie in $B$, so $A \cap B \neq \emptyset$. (1) implies $f: A \cap B \rightarrow A \cap B$ and (2) implies that $f$ restrected to $A \cap B$ is a probabilistic contraction mapping. By theorem 2.1 with $\varphi(t)=k t$, $f$ has a unique fixed point in $A \cap B$.

Corollary 3.1. Let $A$ and $B$ be two non-empty closed subsets of a complete probabilistic metric space $\left(M, F, \tau_{T}\right)$. Let $f: A \rightarrow B$ and $g: B \rightarrow A$ be two functions such that

$$
F_{f p g q}(k t) \geq F_{p q}(t) \forall p \in A \text { and } \forall q \in B
$$

where $k \in(0,1)$. Then there exists a unique $r \in A \cap B$ such that

$$
f(r)=g(r)=r .
$$

Proof. Apply theorem 3.1 to the mapping $h: A \cup B \rightarrow$ $A \cup B$ defined by

$$
h(p)=\left\{\begin{array}{lll}
f(p) & \text { if } & p \in A \\
g(p) & \text { if } & p \in B
\end{array}\right.
$$

Observ that the mapping $h$ is well define because if $p \in A \cap B$, (3.2) implies

$$
F_{f p g p}(t) \geq F_{p p}\left(\frac{t}{k}\right) \text { for all } t>0
$$

Then $F_{f p g p}=\epsilon_{0}$, so $f(p)=g(p)$
The reasoning of Theorem 3.2 can be extended to a colletion of finite sets.

Theorem 3.3. Let $\left\{A_{i}\right\}_{i=1}^{m}$ be nonempty closed subsets of a complete probabilistic metric space, and suppose $f: \bigcup_{i=1}^{m} A_{i} \rightarrow \bigcup_{i=1}^{m} A_{i}$ satisfies the following conditions (where $\left.A_{p+1}=A_{1}\right)$ :

(1) $F\left(A_{i}\right) \subset A_{i+1}$ for $1 \leq i \leq p$;

(2) $\exists k \in(0,1)$ such that $F_{f p f q}(k t) \geq F_{p q}(t) \forall p \in A_{i}$, $\forall q \in A_{i+1}$ for $1 \leq i \leq p$.

Then $f$ has a unique fixed point in $\bigcap_{i=1}^{m} A_{i}$.

Proof. Let $p_{0} \in \bigcup_{i=1}^{m} A_{i}$, we observe that, infinitely terms of the Cauchy sequence $\left\{p_{n}\right\}$ lie in each $A_{i}$. Thus $\bigcap_{i=1}^{m} A_{i} \neq \emptyset$, and the restriction of $f$ to this intersection is a probabilistic contraction mapping. By theorem $2.1 f$ has a unique fixed point in $\bigcap_{i=1}^{m} A_{i}$.

Conflict of Interest The authors declare that they do not have any competing interests.

\section{References}

[1] K. Menger, Statistical metrics, Proc. Natl. Acad. Sci. 28 (1942), 535-537.

[2] W. A. Kirk, P. S. Srinivasan, P. Veeramani, Fixed points for mappings satisfying cyclical contractive conditions. Fixed Point Theory, volume 4, No. 1, 2003, 79-89.

[3] Schweizer B. and A.Sklar, Probabilistic Metric Spaces, NorthHolland Series in Probability and Applied Mathimatics, 5, (1983).

[4] O. Hadzić, A fixed point theorem in Menger spaces, Publ. Inst. Math. (Beograd) T 20 (1979) 107-112. http://eudml.org/doc/257517

[5] A. Mbarki, A. Benbrik, A. Ouahab, W. Ahid and T. Ismail, Comments on "Fixed Point Theorems for $\varphi$-Contraction in Probabilistic Metric Space", Int. J. Math. Anal. 7, 2013, no. 13, 625 - 635. doi 10.12988/ijma.2013.13060

[6] L. Ciric, Solving the Banach fixed point principle for nonlinear contractions in probabilistic metric spaces, Nonlinear Analysis 72 (2010) 2009-2018. doi 10.1016/j.na.2009.10.001 